\title{
1D-2D-3D transition conditions in transient IR thermographic NDE
}

\author{
by V. P. Vavilov \\ Tomsk Polytechnic University, Russia, 634028, Tomsk, 28, Savinykh St., 3 \\ Tel.+7(3822)418712 Fax+7(3822)417281 E-mail: vavilov@introscopy.tpu.ru
}

\begin{abstract}
Lateral heat diffusion phenomena are discussed by using 1D, 2D and 3D models for solids with hidden defects. Critical values of the ratio between defect lateral size and defect depth are obtained to specify the transition to a 1D case. The three techniques for determining defect lateral dimensions and configuration are evaluated.
\end{abstract}

\section{Introduction}

It is well known that lateral heat diffusion in solids with hidden defects reduces temperature signals over defects and shortens their observation times. However, there is still a lack of the reliable data on what geometrical and thermal conditions specify the transition from finite defects into infinite. The available remarks that, first, a defect lateral size should be at least twice as a defect depth, and, second, a temperature profile exhibits a size shrinkage, are not detailed enough to choose between 1D, 2D or 3D geometries [1,2].

In this study, we intend to finalize, up to some extent, the recommendations on choosing a proper thermal NDT model (see also [3]). The involved parameters of the studied models are: 1) heating time, 2) observation time, 2) sample thickness and thermal properties, 3) defect lateral size, thickness and thermal properties.

The analysis has been conducted by applying the Multilayer-3 and ThermoCalc-2D, 3D software that is being used for years in Tomsk Polytechnic University in modeling thermal NDT problems.

A 1D Cartesian model is analytical and presents a three-layer plate where a central layer can subsequently simulate either a bonding layer or a defect (Multilayer-3 program). A 2D cylindrical model is numerical and models a three-layer disk with a disk-shaped defect (ThermoCalc-2D program). The typical number of grid nodes is $50 \times 100=5000$. Finally, a 3D Cartesian numerical model simulates a three-layer parallelepiped that can contain up to six parallelepiped-like defects (ThermoCalc-3D program). The number of grid nodes has been typically about $25 \times 25 \times 100=62500$, although up to one million nodes might be involved in some cases. It has been possible to simulate heating with a flash, square- or cosine-pulse, or harmonic function. In this paper, all results relate to a practical case of square-pulse heating.

\section{When a disk-shaped defect transits into a laterally-infinite layer?}

In many samples, the hidden defects, such as delaminations or disbonds, can be modeled with a thin disk of the radius $r_{d}$. In transient thermal NDT, a dynamic temperature distribution over a defect is typically bell-shaped. Let us imagine that the defect radius is enlarging laterally. Obviously, the temperature signal over the defect $\Delta T$ will grow up to a certain limit. This limit will specify the transition to a 1D model (within this model, edge phenomena are neglected, and $\Delta T$ is constant over a vast defect area). Here it is worth to refer to the known 
rule of thumb which states that a $\Delta T$ signal decreases dramatically if $r_{d} /<1$. In this section we shall put this intuitive rule on a more quantitative basis.

Following the approach described in [4], let us characterize lateral heat diffusion with the coefficient $k_{\text {dif }}=\Delta T\left(r_{d} / l, \tau\right) / \Delta T\left(r_{d} / l \rightarrow \infty, \tau\right)$. Its values for the three different observation times $\tau_{1}, \tau_{2}, \tau_{3}$ are presented in figure 1 for a $5 \mathrm{~mm}$ graphite/epoxy composite sample.

The $r_{d} / l$ values that specify the $2 \mathrm{D} \rightarrow 1 \mathrm{D}$ transition are presented in table 1 for $\Delta T_{2 D} \geq 0.9 \cdot \Delta T_{1 D}$ as shown in figure 1 (here the subscripts "1D" and "2D" specify a $1 \mathrm{D}$ and 2D case). Notice that, in fact, a cylindrical 2D geometry represents a practical 3D case.

The data in table 1 are given for the three practical NDT cases: 1) heating a $2 \mathrm{~mm}$ aluminum sample with a flash, 2) heating a $5 \mathrm{~mm}$ graphite/epoxy composite sample with a flash, and 3) heating a $20 \mathrm{~cm}$ soil layer with a periodical solar radiation (the latter case might simulate detecting buried mines). Defect depths and thicknesses are given in percent of a sample thickness (10\% and $30 \%)$.

The main conclusions from table 1 are as follows.

- Critical $r_{d} / l$ values are greater than it is typically expected. In fact, this ratio is in the range $r_{d} / l>3 \div 17$ depending on the situation.

- Materials of higher conductivity are characterized with greater $r_{d} / l$ critical values.

- Deeper defects require smaller $r_{d} / l$ critical values.

- Thicker defects require greater $r_{d} / l$ critical values.

Table 1

Approximate critical $r_{d} / l$ values specifying transition between 2D (cylindrical) and 1D thermal NDT model by $90 \%$ of a maximum $\Delta T$

\begin{tabular}{|c|c|c|}
\hline Defect depth $l$ & Defect thickness $d$ & Critical $\left(r_{d} / l\right)_{c r}$ values \\
\hline \multicolumn{3}{|c|}{ Aluminum: $K=210 \mathrm{~W} /(\mathrm{m} \mathrm{K}), \alpha=8.6 \cdot 10^{-5} \mathrm{~m}^{2} / \mathrm{s}, L=2 \mathrm{~mm}, Q=10^{5} \mathrm{~W} / \mathrm{m}^{2}, \tau_{h}=0.01 \mathrm{~s}$} \\
\hline \multirow[t]{2}{*}{$10 \% L(0.2 \mathrm{~mm})$} & $10 \% L(0.2 \mathrm{~mm})$ & 15 \\
\hline & $30 \% L(0.6 \mathrm{~mm})$ & 17 \\
\hline \multirow[t]{2}{*}{$30 \% L(0.6 \mathrm{~mm})$} & $10 \% L(0.2 \mathrm{~mm})$ & 7 \\
\hline & $30 \% L(0.6 \mathrm{~mm})$ & 7 \\
\hline \multicolumn{3}{|c|}{ Graphite/epoxy composite: } \\
\hline \multicolumn{3}{|c|}{$K=0.64 \mathrm{~W} /(\mathrm{mK}), \alpha=5.2 \cdot 10^{-7} \mathrm{~m}^{2} / \mathrm{s}, L=5 \mathrm{~mm}, Q=10^{5} \mathrm{~W} / \mathrm{m}^{2}, \tau_{h}=0.01 \mathrm{~s}$} \\
\hline \multirow[t]{2}{*}{$10 \% L(0.5 \mathrm{~mm})$} & $10 \% L(0.5 \mathrm{~mm})$ & 5 \\
\hline & $30 \% L(1.5 \mathrm{~mm})$ & 7 \\
\hline \multirow[t]{2}{*}{$30 \% L(1.5 \mathrm{~mm})$} & $10 \% L(0.5 \mathrm{~mm})$ & 3 \\
\hline & $30 \% L(1.5 \mathrm{~mm})$ & 5 \\
\hline \multicolumn{3}{|c|}{ Soil: $K=1.1 \mathrm{~W} /(\mathrm{mK}), \alpha=7.5 \cdot 10^{-7} \mathrm{~m}^{2} / \mathrm{s}, L=20 \mathrm{~cm}, Q=10^{3} \mathrm{~W} / \mathrm{m}^{2}, \tau_{h}=12 \mathrm{hr}$} \\
\hline \multirow[t]{2}{*}{$10 \% L(2 \mathrm{~cm})$} & $10 \% L(2 \mathrm{~cm})$ & 8 \\
\hline & $30 \% L(6 \mathrm{~cm})$ & 9 \\
\hline \multirow[t]{2}{*}{$30 \% L(6 \mathrm{~cm})$} & $10 \% L(2 \mathrm{~cm})$ & 4 \\
\hline & $30 \% L(6 \mathrm{~cm})$ & 4 \\
\hline
\end{tabular}




\section{Early detection principle. Determining defect lateral size.}

It is well seen from figure 1 that the $k_{d i f}\left(r_{d} / l, \tau\right)$ dependence is steeper for shorter times. This means that, at shorter times, lateral heat diffusion around hidden defects is weaker than at longer times. The corresponding early detection technique is able to ensure a defect image quality similar to that obtained with ultrasonic [5].

The next problem to be discussed is how to reconstruct a configuration (true lateral dimensions) of a hidden defect by its surface 'footprint'. There are three techniques to do this: 1) establishing a particular $\Delta T_{\text {thr }}$ threshold value over a sound level, 2) measuring the contour corresponding to a $\Delta T_{m} / 2$ level (this technique calls FWHM - full-width at halfmaximum measurements), and 3) measuring the contour corresponding to extremums in the first temperature derivative $(d T / d x)_{\text {extr }}$ or to zero in the second temperature derivative $\left(d^{2} T / d x^{2}\right)_{\text {zero }}$.

The first approach is more or less intuitive but noise and time dependent. We found that it can produce both under- and over-estimate of defect true dimensions.

The second approach has been discussed within the shrinkage concept [2]. The main conclusion is that a defect size estimate depends on time and requires travelling to a zero time when estimates are most accurate.

Finally, it has been suggested that the third technique is stable against all involved parameters and can produce the most reliable result [6]. However, the need to compute a signal slope enhances noise and makes this technique difficult in case of small defects.

The results of applying all three reconstruction techniques are presented in table 2. The first technique relates to the threshold $\Delta T_{t h r}$. Because of its dependence on the excess temperature (absorbed energy) and time, this technique proved to be unreliable although it can be used in detecting defects. The FWHM technique produces acceptable results with a typical accuracy not worse than 15\% (except too long observation times in high-conductive metals) and can be recommended in practice. Notice that the phenomenon of shrinkage was not observed in the analyzed situations in a pure form. The third technique requires analyzing the extremums of the first surface derivative $(d T / d x)_{\text {extr }}$ that appears as a maximum in the ascending $\Delta T$ slope and as a minimum in the descending $\Delta T$ slope [6]. Respectively, the second derivative is zero in both slopes. In all analyzed cases, the reconstructed defect radius values were exactly equal to the true values with the accuracy being within one spatial grid step in a radial direction. The difficulty in applying the $(d T / d x)_{\text {extr }}$ technique is that each derivative operation enhances noise.

Reconstructing defect lateral size by analyzing surface spatial profiles

\begin{tabular}{|c|c|c|c|c|}
\hline \multirow{2}{*}{$\begin{array}{c}\text { Observation } \\
\text { time, }\end{array}$} & \multirow{2}{*}{$\begin{array}{c}\text { True defect } \\
\text { radius, mm }\end{array}$} & \multicolumn{3}{|c|}{ Reconstructed defect radius, mm } \\
\cline { 3 - 5 } & & by $(d T / d x)_{\text {extr }}$ & by $\Delta T_{m} / 2$ & by \\
& & & $\Delta T_{t h r}=0.01^{\circ} \mathrm{C}$ \\
\hline \multicolumn{3}{|c|}{ Aluminum: $L=2 \mathrm{~mm}, l=0.5 \mathrm{~mm}, d=0.2 \mathrm{~mm}, Q=10^{6} \mathrm{~W} / \mathrm{m}^{2}, \tau_{h}=0.01 \mathrm{~s}$} \\
\hline 0.005 & \multirow{3}{*}{1.0} & $1.0^{*}$ & 1.0 & 2.5 \\
\hline 0.01 & $1.0^{*}$ & 1.0 & 3.0 \\
\hline 0.05 & $1.0^{*}$ & 2.4 & 1.8 \\
\hline
\end{tabular}




\begin{tabular}{|c|c|c|c|c|}
\hline \multicolumn{5}{|c|}{ Graphite/Epoxy Composite: } \\
\hline \multicolumn{5}{|c|}{$L=5 \mathrm{~mm}, l=1.5 \mathrm{~mm}, d=0.5 \mathrm{~mm}, Q=10^{5} \mathrm{~W} / \mathrm{m}^{2}, \tau_{h}=0.01 \mathrm{~s}$} \\
\hline \multirow{2}{*}{5.0} & $5.0^{*}$ & 5.4 & 4.0 \\
\cline { 3 - 5 } & \multirow{2}{*}{5.5} & $5.0^{*}$ & 4.6 & 7.0 \\
\hline 5.0 & & $5.0^{*}$ & 4.3 & 7.5 \\
\hline
\end{tabular}

* By accuracy of one spatial grid step in a radial direction

\section{3D model and defect lateral configuration}

The new information that can be obtained within a 3D model compared to the proper 2D model is how a signal $\Delta T$ over a defect is affected by a defect configuration.

By using the ThermoCalc-3D program we have analyzed several defects in a graphite/epoxy sample (see figure 2 and table 3). In figure 2, from left to right, the temperature distributions over five $100 \mathrm{~mm}^{2}$ defects are presented (the defects 1-4 are rectangular, the defect 5 is circular). The next two defects $6(20 \times 20 \mathrm{~mm})$ and $7(40 \times 40 \mathrm{~mm})$ clearly represent the 1D case. The strongly 3D cases are modeled with the defects 8 $(2.5 \times 2.5 \mathrm{~mm})$ and $9(5 \times 5 \mathrm{~mm})$. It is seen that, due to lateral heat diffusion, the extended but thin defect $1(50 \times 2 \mathrm{~mm})$ produces only $50 \%$ of the signal above the $10 \times 10 \mathrm{~mm}$ defect 4 with the optimum observation time being also reduced by about $50 \%$. It is interesting that the same signal reduction appears for the smallest defect 8 . Generally, both square-shaped and circle-shaped defects produce close maximum signals at about the same observation times.

Table 3

Influence of defect configuration on surface temperature signal parameters (graphite/epoxy composite: $L=5 \mathrm{~mm}, l=1 \mathrm{~mm}, d=0.5 \mathrm{~mm}, Q=10^{5} \mathrm{~W} / \mathrm{m}^{2}, \tau_{h}=0.01 \mathrm{~s}$;

ThermoCalc-3D software)

\begin{tabular}{|c|c|c|c|}
\hline Defect number & Defect area, $\mathrm{mm}$ & $\Delta T_{m},{ }^{o} \mathrm{C}$ & $\tau_{m}, s$ \\
\hline 1 & $50 \times 2$ & 0.138 & 1.8 \\
\hline 2 & $25 \times 4$ & 0.224 & 2.0 \\
\hline 3 & $12,5 \times 8$ & 0.274 & 2.9 \\
\hline 4 & $10 \times 10$ & 0.277 & 3.0 \\
\hline $5 *$ & Circle $100 \mathrm{~mm}^{2}$ & 0.266 & 2.9 \\
\hline 6 & $20 \times 20$ & 0.279 & 3.0 \\
\hline 7 & $40 \times 40$ & 0.279 & 3.0 \\
\hline 8 & $5 \times 5$ & 0.132 & 1.6 \\
\hline 9 & $2.5 \times 2.5$ & 0.219 & 2.0 \\
\hline
\end{tabular}

* This case was computed by using ThermoCalc-2D software

\section{Special 3D cases}

3D models also allow analyzing the phenomena of uneven heating and defect overlapping. This topic has been thoroughly discussed in earlier publications (see, for instance, [7]). Here we illustrate it with the data in figure 3. The four strip-like defects are located at different depths in a steel sample being laterally overlapped. The color thermograms made in the Iron palette clearly illustrate the known fact that the shallow defect, in its signal time evolution, becomes visible first, even if the heating is uneven (more powerful in the bottom left corner of the sample presented in figure 3). The footprints of the deeper defects appear at later times and experience strong edge blurring. Obviously, a temperature signal is higher in the areas where the defects are laterally overlapped. It has been found that an overall temperature distribution $\Delta T_{\sum}(x, y, \tau)$ can be represented as the 
superposition of the signals from the small-size defects both spatially and temporally: $\Delta T_{\sum}(x, y, \tau)=\sum \Delta T_{i}(x, y, \tau)$. Nevertheless, we still believe that distinguishing between deeper and shallower sections of overlapped defects is scarcely possible because of the subtle difference that these sections bring into the overall spatial and temporal distributions.

\section{Conclusions}

- Analytical 1D heat conduction solutions, particularly, in case of multi-layered structures, are bulky but illustrative allowing the analysis of how defect depth and thermal properties influence on a surface temperature signal $\Delta T$. However, lateral heat diffusion is not negligible in many practical cases thus requiring to solve $2 \mathrm{D}$ and $3 \mathrm{D}$ problems. $\mathrm{A} 2 \mathrm{D}$ cylindrical model is still a good compromise between the model identity and computing time. It typically involves 5000-1000 numerical grid nodes. Within this model, where a disk-shaped defect is placed in a disk-shaped sample, it has been demonstrated that a finite-size defect transits into the corresponding extended 1D defect if a defect radius $r_{d}$ is much larger than its depth $l$. The needed ratio $r_{d} / l$ depends on material, time, defect depth and thickness and exceeds the values predicted by the known rule of thumb.

- 3D heat conduction solutions involve 50000-100000 numerical grid nodes but allow analyzing a few phenomena that are impossible to study in simpler models. These phenomena are: 1) lateral defect configuration, 2) uneven heating and 3) signal superposition over overlapped defects.

- For a constant defect lateral area, maximum signals are created by disk-like defects, meanwhile long but thin defects cause serious reducing of $\Delta T$. The same conclusion relates to optimum observation time.

- In case of laterally overlapped small defects, both spatial and temporal temperature signal distributions can be determined by using the principle of signal superposition. However, detecting a deeper defect behind a shallower one is scarcely possible in a practical test.

\section{References}

[1] MALDAGUE (X.) - Nondestructive evaluation of materials by infrared thermography. Springer-Verlag, Germany, 1993.-208 p.

[2] ALMOND (D.P.), SAINTEY (M.B.) and LAU (S.K.) - Edge-effects and defect sizing by transient thermography. In: Proc. Eurotherm Seminar 42 "Quant. InfraRed Thermography QIRT'94", Aug.23-26, 1994, Sorrento, Italy, p.247-252.

[3] VAVILOV (V.P.) - Three-dimensional analysis of transient thermal NDT problems by data simulation and processing. In Proc. SPIE "Thermosense-XXII", vol.4020, p.152-160.

[4] VAVILOV (V.), GRINZATO (E.), BISON (P.G.), MARINETTI (S.) and BALES (M.) Surface Transient Temperature Inversion for Hidden Corrosion Characterization: Theory and Applications. Intern J. Heat and Mass Transfer., vol. 39, No.2,1996, p. 355-371.

[5] KRAPEZ (J.-C.) and BALAGEAS (D.L.) - Early detection by stimulated infrared thermography. Comparison with ultrasonics and holo/shearography. In: Advances in Signal Process. for NDE of Materials, ed. by X.Maldague, NATO ASI Series, Series E: Applied Sciences, Kluwer Academic Publishers, vol.262, p.303-321.

[6] VAVILOV (V.) and SHIRYAEV (V.) - Determining defect lateral size in thermal NDT. Sov.J.NDT ("Defectoscopiya”), 1979, No.11, p.103-106.

[7] BENDADA (A.), MAILLET (D.) and DEGIOVANNI (A.) - Nondestructive transient thermal evaluation of laminated composites: discrimination between delaminations, thickness variations and multidelaminations. In: Proc. Eurotherm Seminar 27 "Quant. Infrared Thermography QIRT'92”, July 7-9, 1992, Châtenay-Malabry, France, p.218-223. 


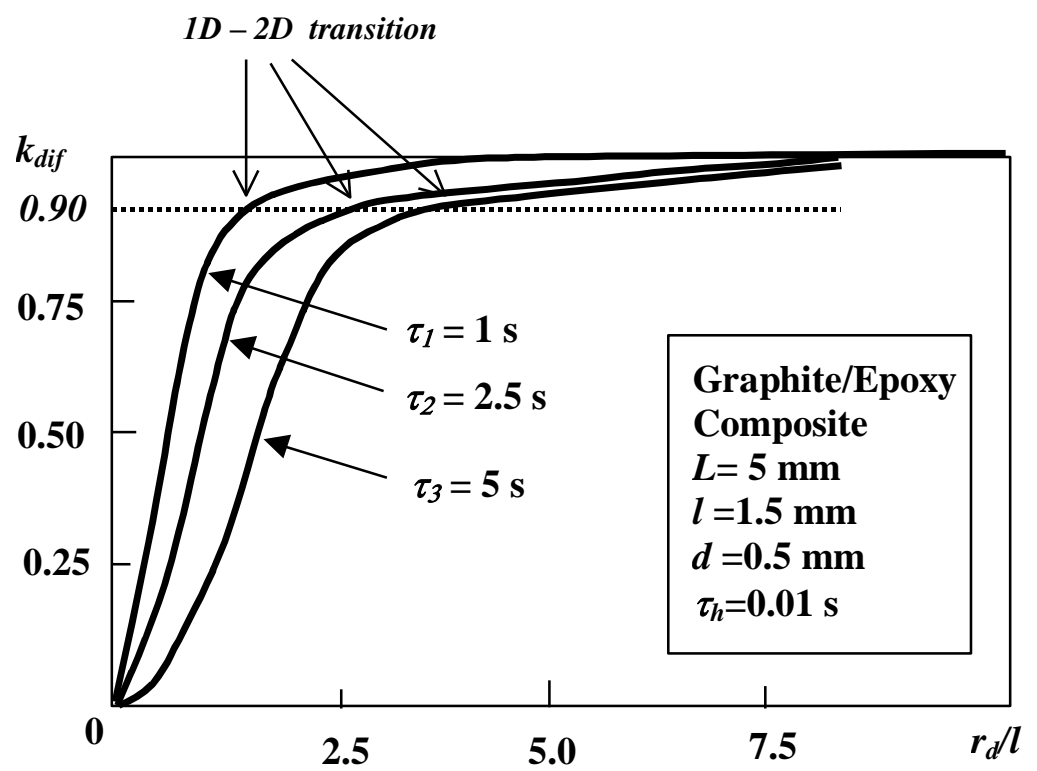

Fig.1. Lateral diffusion coefficient vs. $r_{d} / l$ ratio

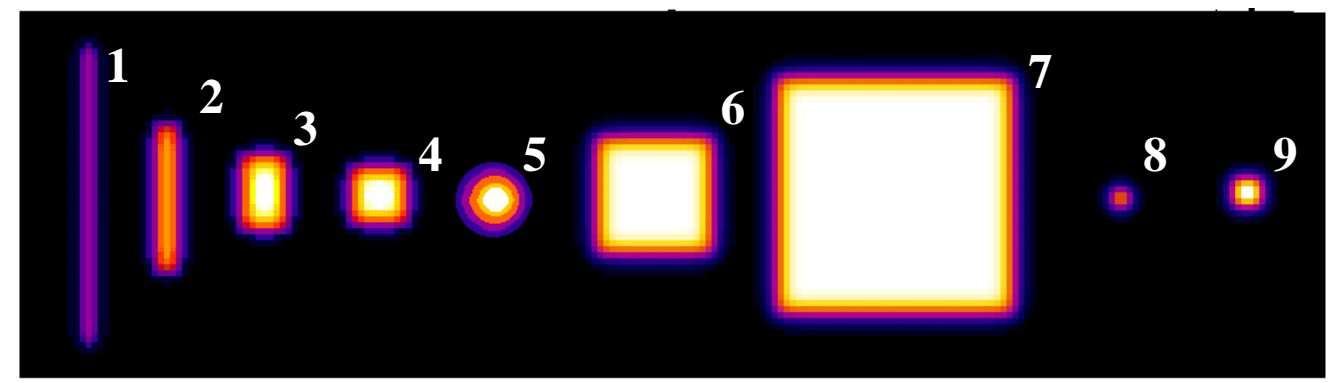

Fig.2. Influence of defect configuration on surface temperature signal appearance (graphite/epoxy composite: $L=5 \mathrm{~mm}, l=1 \mathrm{~mm}, d=0.5 \mathrm{~mm}, Q=10^{5} \mathrm{~W} / \mathrm{m}^{2}, \tau_{h}=0.01 \mathrm{~s}$ )
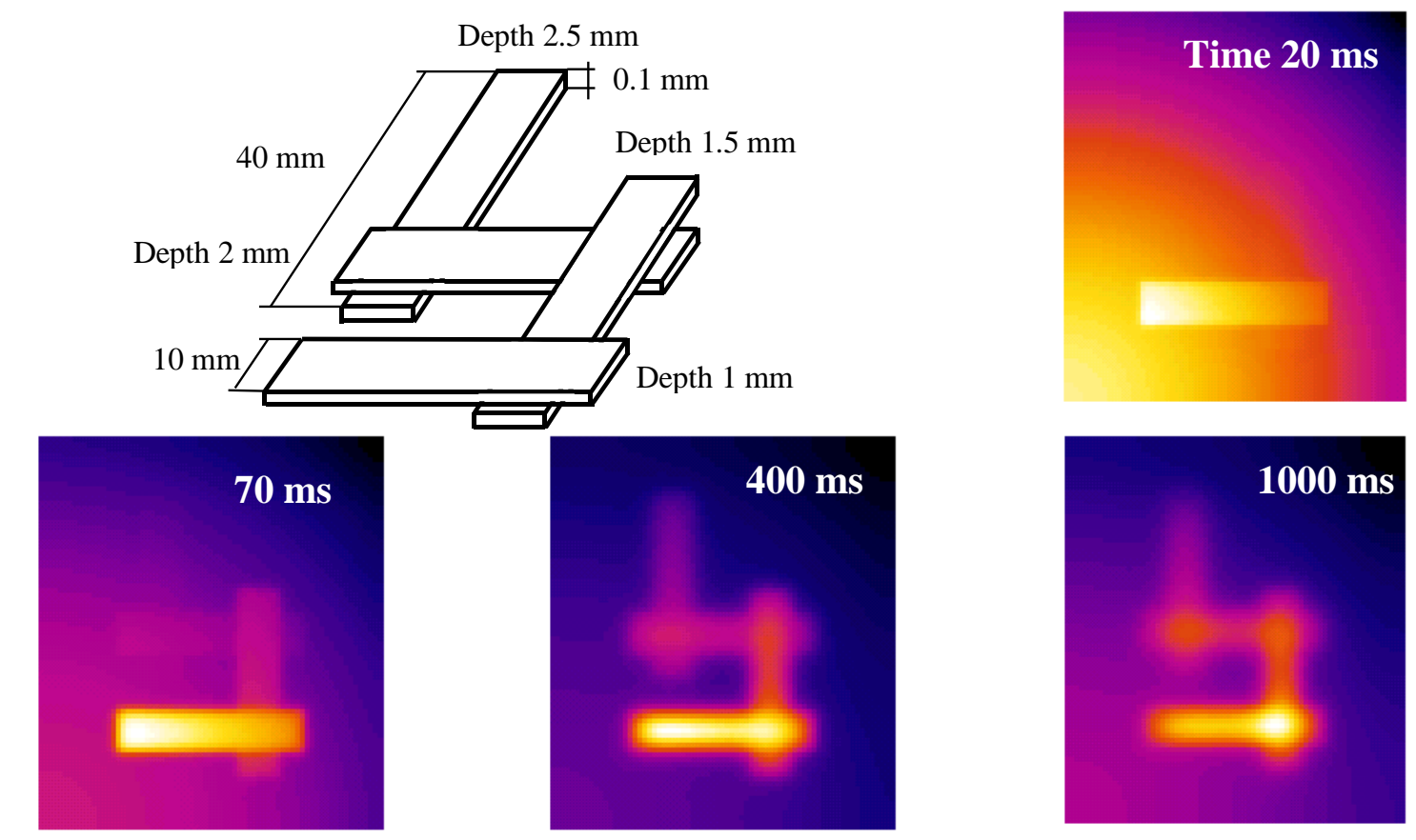

Fig.3. Temperature distributions over four air-filled defects that are located at different depths in a $5 \mathrm{~mm}$ steel AISI-1010 sample and partially overlapped in-plane (heat pulse duration $10 \mathrm{~ms}$ ) 